\title{
1 Recent advances on properties and utility of nanomaterials generated from 2 industrial and biological activities
}

3

\author{
Virendra Kumar Yadav ${ }^{1,2}$, Parth Mallik ${ }^{2}$, Priti Pandita ${ }^{3}$, R. Suriyaprabha ${ }^{2}$, Samreen Heena \\ Khan $^{2}$, Nisha Choudhary ${ }^{2}$, Shreya Modi ${ }^{2}$
}

\author{
${ }^{1}$ School of Lifesciences, Jaipur National University, Jaipur, Rajasthan-302017, India \\ ${ }^{2}$ School of Nanosciences, Central University of Gujarat, Gandhinagar, Gujarat-382030, India \\ ${ }^{3}$ School of Environment and Sustainable Development, Central University of Gujarat-382030, \\ India
}

\section{Abstract}

Today is the era of nanoscience and nanotechnology, which finds applications in the field of medicine, electronics, or environmental cleanup. Even though the nanotechnology is in its emerging phase, but still it provides solutions to numerous challenges. Nanotechnology and nanoparticles are found very effective because of their unique chemical and physical properties, high surface area, but their high cost is one of the major hurdles in its wider application. So, the synthesis of nanomaterials especially 2D nanomaterials from the industrial, agricultural and other biological activities could provide a cost-effective technique. The nanomaterials synthesized from such waste not only minimizes the pollution but also provides an eco-friendly approach towards the utilization of the waste. In the present review work, the emphasis has been given on the types of nanomaterials, different methods for the synthesis of $2 \mathrm{D}$ nanomaterials from the waste generated from industries, agriculture and their application in electronics, medicine and catalysis.

Keywords: Nanomaterials; Carbon nanotubes; Rice husk; Agriculture waste; Carbon nanofibres 


\section{Abbreviations}

27 0-D: 0-dimensional

28 1-D: 1-dimensional

29 2-D: 2-dimensional

30 3-D: 3-dimensional

31 AFM: atomic force microscopy

$32 \mathrm{AgNO}_{3}$ : silver nitrate

$33 \mathrm{Al}_{2} \mathrm{O}_{3}$ : aluminum oxide

$34 \mathrm{BaO}$ : barium Oxide

35 BET: brunauer emitter teller

$36{ }^{0} \mathrm{C}$ : degree celsius

$37 \quad \mathrm{Ca}$ : calcium

$38 \mathrm{CaO}$ : calcium Oxide

39 Cd: cadmium

40 CO: carbon monoxide

$41 \mathrm{Co}_{3} \mathrm{O}_{4}$ : cobalt (II, III) oxide

42 CNTs: carbon nanotubes

43 Cr: chromium 
44 CVD: chemical vapor deposition

45 DMF: N, N-dimethylformamide

46 Fe: ferrous

$47 \quad \mathrm{Fe}_{2} \mathrm{O}_{3}$ : ferric oxide

$48 \quad \mathrm{Fe}_{3} \mathrm{O}_{4}$ : ferric oxide

49 Ga: gallium

50 GO: grapheme oxide

51 HER: hydrogen evolution reaction

52 HRTEM: high resolution Transmission Electron microscopy

53 InSe: Indium selenide

$54 \quad \mathrm{~K}$ : kelvin

55 LCVD: laser chemical vapor deposition techniques

56 LEDs: light emitting diodes

57 Mg: magnesium

$58 \mathrm{MgO}$ : magnesium oxide

$59 \mu \mathrm{m}$ : micrometer

60 Mn: manganese

$61 \mathrm{MnO}$ : manganese oxide 
62 Mo: molybdenum

$63 \mathrm{MoO}_{3}$ : molybdenum trioxide

$64 \mathrm{MoS}_{2}$ : molybdenum disulfide

65 MWCNT: multi walled carbon nanotubes

66 Na: sodium

67 Ni: nickel

68 Nm: nanometre

69 NMs: nanomaterials

70 NSMs: nano structured materials

71 NSs: nanosheets

72 NPs: nanoparticles

73 NSs: nanostructures

74 ORR: oxygen reduction reaction

$75 \quad \mathrm{~Pb}$ : lead

76 Pd: palladium

77 PEM: polymer electrolyte material

$78 \quad \mathrm{P}_{2} \mathrm{O}_{5}$ : phosphorus pentoxide

79 Pt: platinum 
80 PVP: polyvinyl pyrrolidone

81 QD: quantum dots

82 Rice Husk: rice husk

83 SB: sugarcane bagasse

84 Se: selenium

85 SEM: scanning Electron microscopy

86 SERS: surface enhanced Raman scattering

$87 \quad \mathrm{SiO}_{2}$ : silicon dioxide (silica)

88 SnO: stannous oxide

$89 \quad \mathrm{SnO} 2$ : stannic oxide

90 SPR: surface plasmon resonance

91 SQUID: superconducting quantum interface device

92 SS: standard size

93 SVR: surface volume ratio

94 TEM: transmission electron microscopy

95 TGA: Thermo gravimetric analysis

$96 \mathrm{TiO}_{2}$ : Titanium dioxide

97 TPPs: thermal power plants 
$98 \quad \mathrm{WO}_{3}$ : Tungsten trioxide

$99 \mathrm{WS}_{3}$ : Tungsten (VI) Sulfide

100 XRD: $x$-ray diffraction

101 Zn: zinc

102 ZnO: zinc oxide

103 ZnS: zinc sulfide

104

105

106

107

108

109

110

111

112

113

114 
116

117

118

119

120

121

122

123

124

125

126

127

128

129

130

131

132

133

134

135

136

137

\section{Introduction}

Nanotechnology deals with the design and development of the materials at the nanoscale (1-100 $\mathrm{nm}$ ) or one dimension in the nanoscale [1]. The word nano was derived from the Greek word meaning "dwarf" [2] and denoted as nm. By using such measurement, the size of viruses are about $100 \mathrm{~nm}(30-100) \mathrm{nm}$ [3] and to that of a human hair is $1000 \mathrm{~nm}$ in diameter. Nanotechnology and nanoscience allow the researchers to manipulate the properties of materials at the atomic level [4]. Nanomaterials can be produced in a variety of methods like chemical, physical and biological with different classes such as: carbon-based nanomaterials [5], nanocomposites [6], metals, alloys, nanopolymers [7], nanoglassses [8] and nanoceramics [7].

Nanomaterials are typically those materials having at least anyone dimension at the nanoscale $(<100 \mathrm{~nm})$. Nanomaterials can be either synthesized in the laboratory or could be derived from the natural resources [9]. The nanomaterial synthesized from the commercial precursor materials makes the product as well as process expensive. Moreover, the source of nanomaterial is also depleting, so there is a need to rely on the natural and alternative sources of nanomaterial. The natural nanomaterial [10] act as a potential candidate for the development of nanomaterials. The nanomaterial derived from such processes are cost-effective [11], biocompatible [12] and environment friendly [13]. The waste materials that are commonly used for nanomaterial synthesis includes industrial waste like fly ash [14], red mud, agricultural waste [15] (rice husk and straw, wheat husk and straw, coconut shell) and plastic waste [16]. Most of these waste materials mainly act as a pollutant to the environment, which are produced in tonnes annually around the globe. The utilization of such products for the synthesis of carbon nanomaterials reduces the pollution from the environment and simultaneously provides an environment-friendly and economical approach. 
138 These nanostructured materials based on their purities can find application in the electronics [17],

139 wastewater treatment [18], medicine [19] and catalysis [19].

\section{Classification of nanostructured materials}

141 Nanostructured materials (NSMs) have gained a huge consideration in fundamental science and

142 technological applications due to their multifunctionality and unique chemical, physical, electronic

143 and magnetic properties at the nanoscale [20]. Like every day new novel nanomaterials are

144 synthesized so the classification is an utmost need for this.

Fig.1 Classification of nanomaterials

152 The density of the state varies considerably for different nanomaterials which are based on the

153 degree of freedom/confinement [21]. Based on the nanostructural elements and their physical and

154 chemical properties; the nanomaterials have been classified into four classes i.e. 0-D, 1-D, 2-D, 155 and 3-D by Pokropivny. 
157 In 0-D material (quantum dot) [QD], there is confinement of electrons in all the three directions

158 [22]. Zero dimensional nanomaterial has gained a huge attention in the field of research and in 159 material based industries [23]. Such material finds applications in the light emitting diodes (LEDs) 160 [24], solar cells [25], single-electron transistors [26], and lasers. The common example of zero 161 dimensional nanomaterial are spheres (including hollow spheres) and nanoclusters [27], quantum 162 dots that includes core-shell QDs also [28], heterogeneous particles arrays, onions [29], and 163 nanolenses.

\subsection{One dimensional nanomaterial (1D-Nanomaterial)}

One dimensional nanomaterial is those materials which are confined in two dimension but free in one dimension [30]. Some of the common examples of 1D nanomaterial are wires, nanowires [31], nanotubes, nanofibres [32], nanobelts [33], nanoribbons [33], nanorods [34] and hierarchical nanostructures. From the last decade, such nanomaterial has gained huge considerations because of their remarkable properties and such a wider applicability in the research and development and material development. Such materials have wider impact in nanoelectronics [35], nanodevices and systems [36], nanocomposite materials [37], and alternative resources of energy. The 1D nanomaterials are the most preferred material for exploring the properties at the nanoscale. It is also used for the investigation of size and dimensionality dependence of functional properties [38].

\subsection{Two-dimensional nanomaterials (2D nanomaterials)}

2D nanomaterials have only one dimension in the nano range while the other two dimensions are out of nano range [39]. 2D Materials are said to be the thinnest materials, which possess the highest surface area. In recent years, not only the synthesis but also the applications of 2D NSMs have drastically dawn attentions in materials research because of their several interesting properties at 
180 the nanoscale. In comparison with bulk materials, two-dimensional (2D) nanomaterials own rare

181 physiochemical assets develops due to their high aspect ratio (SVR) [40], distinctive surface

182 chemical properties, and quantum confinement effect [41]. The 2D NSMs finds applications in

183 sensing materials, photocatalysis, nanocontainers and nanoreactors [28]. Most preferably, the

184 metallic based 2D NSMs have exploited widely in sensing, catalysis, photothermal therapy, 185 surface-enhanced Raman scattering (SERS), bioimaging, and solar cells [42], due to their 186 phenomenal properties. The common examples of 2D nanomaterials are nanoprisms [43], 187 nanoplates [44], nanosheets [45, 46], nanowalls [46], and nanodisks [47].

\subsection{Three Dimensional nanomaterials (3D-Nanomaterials)}

189 The 3D NSMs three dimensional nanomaterials are those materials which have their free 190 dimensions in all the three directions and there is no confinement and limitations [28]. The 191 common examples of three 3D nanomaterials are powders [48], multilayer [49], fibrous and poly 192 crystalline materials [9]. The 3D nanomaterials exhibit have large specific surface area [50] and 193 because of which such nanostructures provide adequate surface absorption sites for the molecules 194 in a small area. The 3D NSMs are extensively used for catalysis in nanomaterials finds applications 195 in the field of catalysis [51], magnetism and for development of electrode material for batteries 196 [52]. Additionally, the porosity in the three dimensions, helps in the easy transport of the molecules.

197 The examples of 3D NMSs are nanoballs (dendritic structures) [53], nanocoils [54], nanocones 198 [28], nanopillers [55] and nanoflowers [55]. 


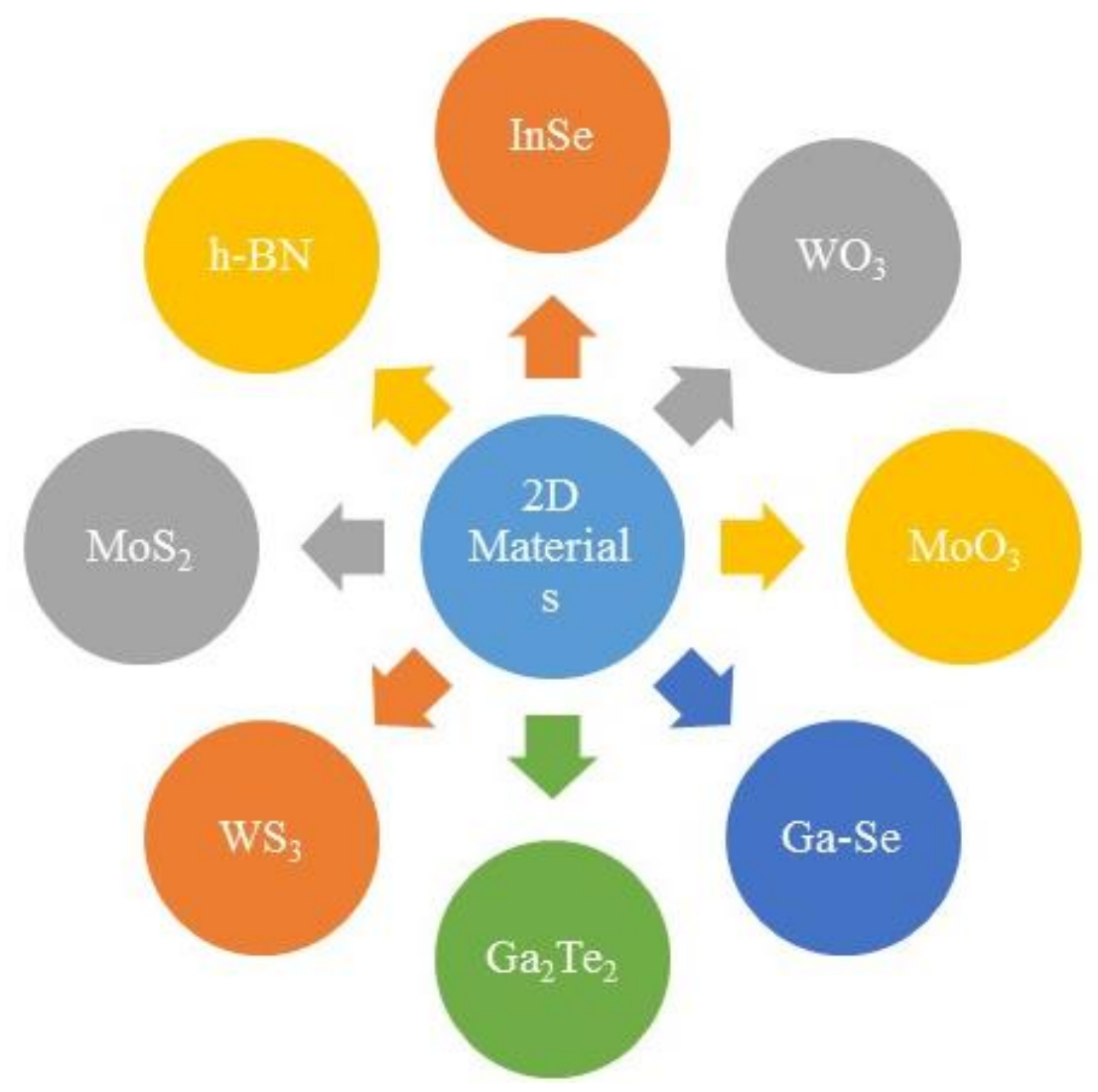

Fig.2 Examples of 2D materials

\section{Different methods of nanomaterials synthesis}

215 The nanomaterials could be developed by all the three means i.e. chemical, physical and biological 216 methods. Among them the physical approaches includes sputtering [56], laser ablation [57], 217 pyrolysis [58], lithography [59], and hot and cold plasma [59]. While, the chemical methods that 218 are used most frequently are lyotropic liquid crystal templates [60], electrochemical deposition 219 [61], electroless deposition [62], hydrothermal [63] and solvothermal techniques [64], sol-gel 220 technique [65, 66], laser chemical vapor deposition technique [67], laser pyrolysis [68] and 221 chemical vapor deposition [69]. The nanomaterials could also be synthesized by the biological 222 approaches like microbial [70] and plant derived materials [71]. The microbial synthesis of 223 nanomaterials [72] employs the utilization of microorganisms like algae [73], fungi [46] and 
224 bacteria [74]. The main drawback is that when there is utilization of commercial precursor for the

225 synthesis of nanomaterials by any of the above mentioned approaches there the process as well as

226 the product become expensive. So, in order to get a cost-effective material the precursor should be

227 lower in cost. One such materials are the industrial waste [75], biological waste or agricultural 228 waste [15].

229

230

231

232

233

234

235

236

237

238

239

240

242

243

244

245

246

247

248

249

250

251

252

253

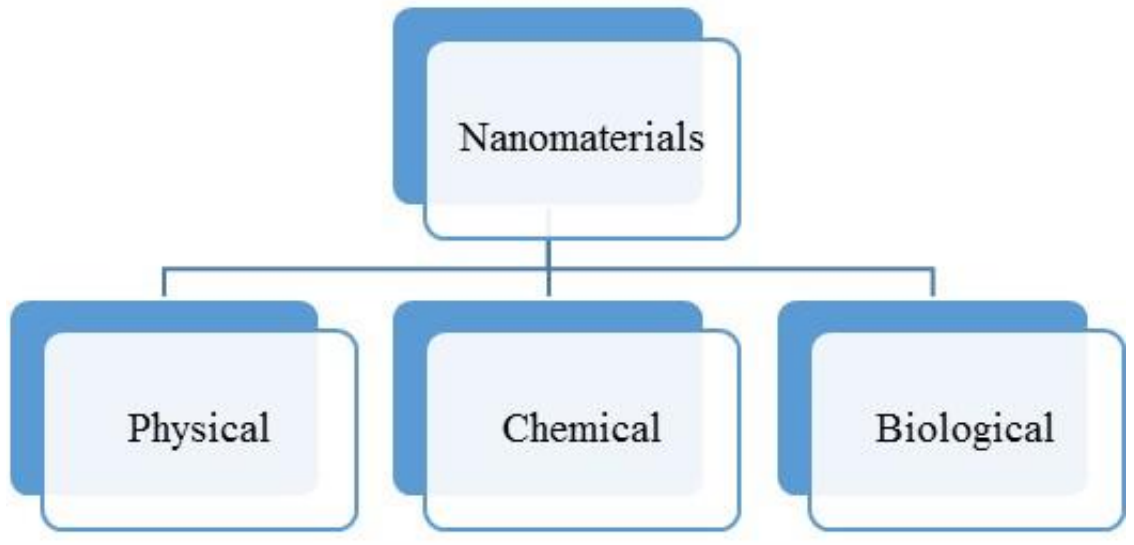

$\angle 41$

Fig. 3 Different methods of synthesis of nanomaterials

\subsection{Physical methods for synthesis of 2D NSMs}

The 2D NSMs could be synthesized by various physical methods [76] such as evaporation [77], lithography [78], sputtering, phase condensation, hot and cold plasma spray pyrolysis [79], inert gas phase condensation [80], pulsed laser ablation method [81] and sonochemical reduction [82]. These methods (physical) are generally used for the synthesis of nanowalls [46], nanoprisms [83], nanosheets [84], nanoplates [85], and nanodisks [28]. The nanomaterials synthesized by physical method are homogenous in nature and ordered. Dai et al., 2002 developed the SnO nanodisks [56]alumina plates using thermal evaporation method under optimized environmental conditions [86].

Here firstly, $\mathrm{SnO}$ or $\mathrm{SnO}_{2}$ powders were kept in an alumina boat which in turn placed in a quartz tube reactor (evaporation source), where alumina act as a substrate which was placed one by one 
254 in the downstream. The physical techniques provides an environment friendly approach for the

255 development of 0D; 1D; 2D; and 3D nanomaterials which are shown below in Figure 5.

256

257

258

259

260

261

262

263

264

265

266

267

268

269

270

271

Fig. 5 Physical methods for the synthesis of 2D nanomaterial

272

\subsection{Chemical methods for synthesis of nanomaterials}

274 Chemical methods have contributed to the fabrication of materials at nanoscale [87]. The Chemical

275 methods have several advantageous properties over physical methods as the previous one involves

276 mixing of chemical at molecular level which ensures good chemical homogeneity [66, 88].

277 Chemical reduction methods are reported to have numerous drawbacks for instance utilization

278 toxic reagents and solvents, generation of unwanted by products due to which there are several 
279 extra step is needed for removal of impurities, time consuming [89]. The most common chemical

280 methods are electroless deposition [90], lyotropic liquid crystal templates [28], hydrothermal and

281 solvothermal method, sol-gel technique, electrochemical deposition, chemical vapor deposition

282 (CVD), laser pyrolysis and laser chemical vapor deposition techniques (LCVD) which are utilized

283 frequently for the production of different NSMs. The above-mentioned techniques are shown in

284 the Figure 6.

285

286

287

288

289

290

291

292

293

294

295

296

297

298

299

Fig. 6 Chemical methods of synthesis of nanomaterials

Among all the metallic nanoparticles silver nanoparticles has gained used consideration due to

301 their exceptional properties and applications. Silver nanoparticles of different shapes and sizes 
302 have important role in medicine, biomedical field and drug delivery [91]. Till now silver NPs of

303 various shapes and sizes has been reported by the numerous investigators. Nanoprisms are one of

304 the example of 2D nanomaterial, which had gained huge attention in the biomedical field [91].

305 Silver nanoprisms were synthesized silver salts by chemical reduction and photochemical method where

306 the earlier method is more preferred than the later one due to their more controlled growth of nanoprisms

307 which finds application in the industries [92]. Monodispersed hematite $\left(\mathrm{a}-\mathrm{Fe}_{2} \mathrm{O}_{3}\right)$ nanodiscs of size

$308(50 \pm 10 \mathrm{~nm}$ in diameter and thickness of $6.5 \mathrm{~nm})$ synthesized under a mild conditions through a

309 facile hydrothermal method i.e. hydrolysis of ferric chloride [93]. The reported method was quite

310 unique as there was no use of surfactants, no toxic or hazardous chemical precursors, no high

311 temperatures decomposition of iron precursors in non-polar solvents. The synthesized hematite

312 nanodiscs were further characterized by the atomic force microscopy (AFM), X-ray diffraction

313 (XRD), Scanning Electron microscopy (SEM), Transmission Electron microscopy (TEM),

314 Brunauer Emitter Teller (BET), and superconducting quantum interface device (SQUID). The

315 synthesis of $\mathrm{Ta}_{3} \mathrm{~N}_{5}$ nanoplates was reported by Jie Fu and Sara E. Skrabalak, 2016, for the

316 photocatalytic application [94]. A simple technique developed for the production of hexagonal-

317 shaped Ag nanoplates whose diameter was in the range of 15-20 nm with a smooth nanobulk of

$318120 \mathrm{~nm}$ [95]. The silver nanoplates were prepared by a kinetically controlled solution growth

319 method under following conditions; polyvinyl pyrrolidone (PVP) as a capping agent, dextrose as

320 reducing agent, and urea as a habit modifier at $50{ }^{\circ} \mathrm{C}$ and the crystalline structure of silver

321 nanoplates analyzed by the XRD and TEM.

322 Xin He et al., 2009 synthesized triangular/hexagonal silver nanoplates, nanobelts and chain-like

323 nanoplate assemblies by utilizing N,N-dimethylformamide (DMF) along with PVP [96]. The

324 results revealed that due to the strong interaction between $\mathrm{Ag}^{+}$and PVP, there was the formation

325 of individual nanoplates and external features of nanoplates were controlled by the ratio of $\mathrm{AgNO}_{3}$ 
326

327

328

329

330

331

332

333

334

335

336

337

338

339

340

341

342

343

344

345

346

347

348

and PVP. Sial et al., 2018 synthesized multimetallic nanosheets which was utilized for the manufacturing of fuel cells [97]. Zheng et al.,2011 synthesized Palladium NSs by using CO as a reducing agent [98]. Yansong Zhou et al., 2016 reported an ultra-facile and generalized approach for the synthesis of metal oxide nanosheets $\left(\mathrm{TiO}_{2}, \mathrm{Co}_{3} \mathrm{O}_{4}, \mathrm{Fe}_{2} \mathrm{O}_{3}, \mathrm{ZnO}\right.$, and $\left.\mathrm{WO}_{3}\right)$ with larger surface and applied them for the for energy applications [99]. Jianxing Liu, He Yang, Xiangxin

Xue 2018, reported the synthesis of hematite nanosheets by using a large sized particles of iron red and found that the shape of hematite have important effect on the magnetic and optical properties [100]. All the above mentioned chemical processes revealed simple, reliable and useful approach towards synthesis of 2D NSMs. The shape, size and composition of the 2D NSMs can be varied by precursor solutions, conditions of deposition and substrate materials [76].

\subsection{Biological methods for the synthesis of 2D NSMs}

Biological synthesis of nanomaterials involves the synthesis from plants and their parts, microbes for instance algae, fungi and bacteria. In comparison to the chemical and physical method biological methods are eco-friendly and there is minimum utilization of hazardous chemicals. Besides this the nanomaterials synthesized by biological methods are biocompatible. There are several reports where the nanomaterials have been synthesized by biological methods [101].

\section{Carbon nanomaterials}

Carbon is not only the most abundant element on earth crust but it also acquires exceptional properties because of its hybrid orbitals. The allotropes of carbon is mainly due to the hybridization of bonds formed after the combination of atomic orbitals ( $\mathrm{s}$ and $\mathrm{p}$ ) into new hybrid orbitals as $s p$, $s p^{2}$, and $s p^{3}$. The different allotropes of carbon are buckyballs (0D), CNTs (1D), graphene sheets (2D), and diamond (3D) [102]. Due to the allotropy, carbon forms a separate class of 2D nanomaterials that includes graphene, GO, CNTs, buckyballs and its derivatives which are shown 
349 in the Figure. 7 and the properties of graphene oxide (GO) is shown in Figure 8. All these 350 nanocarbons finds applications in electronics, environmental cleanup, drug delivery, agriculture, 351 research and catalysis [103]. The wider applications of nanocarbons are also due to presence of 352 wide range of structural and textural properties in them. Out of all, nanocarbons, CNTs and 353 graphene are the most widely used nanomaterials in the field of nanotechnology [103].

354

355

356

357

358

359

360

361

362

363

364

365

366
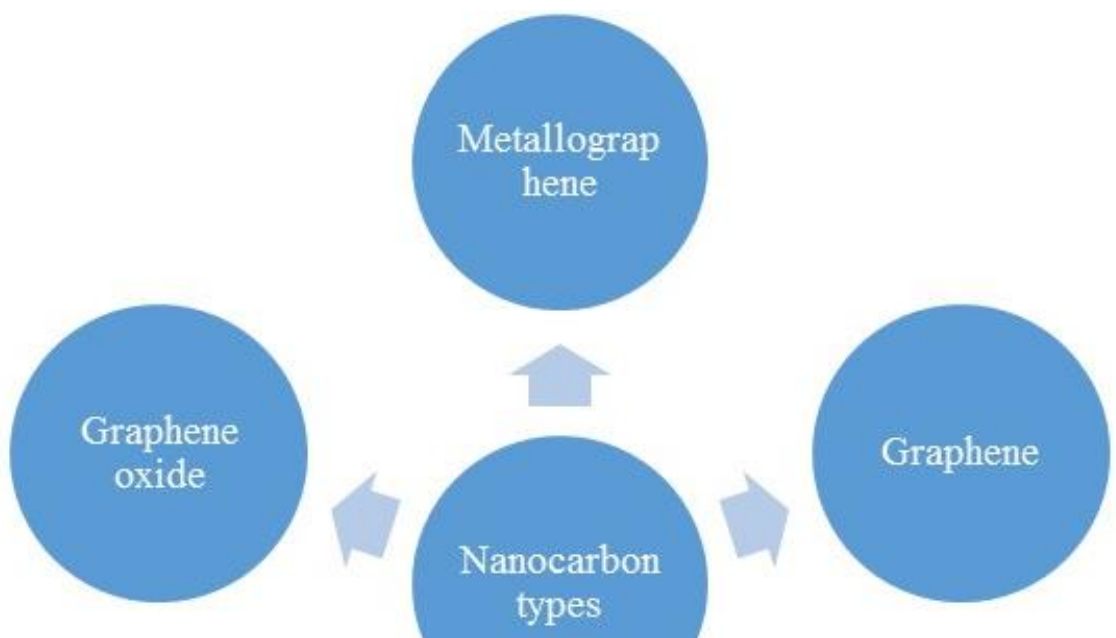

Fig.7 Different types of nanocarbons 


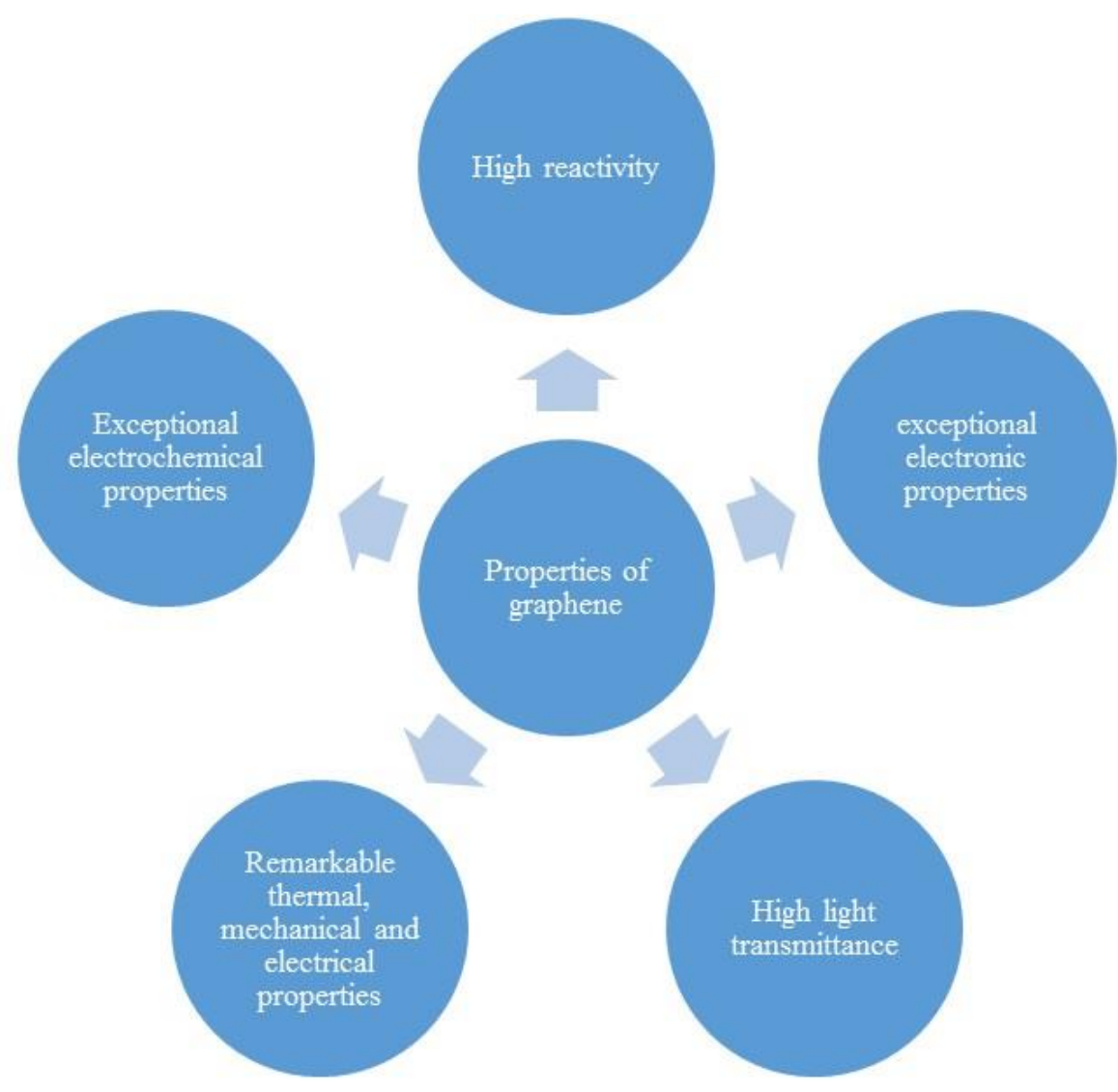

380

Fig. 8 Properties of graphene

\subsection{Synthesis of GO from agro waste}

384 Sugarcane bagasse (SB) [104] is an agricultural waste which is rich in carbon content. Every year 385 it is produced in million tonnes around the whole world and challenges a potential threat for the 386 environment. The recovery of nanocarbons and GO from such waste will reduce the pollution from 387 the environment. The recovery of GO from sugarcane bagasse includes following steps, collection 388 of the fiber, crushing followed by grinding to obtain a powder, repetition of these two steps in 389 order to increase the fineness of the powder [105]. Grounded SB and ferrocene was mixed in 5:1 390 ratio by weight, in a crucible and calcination was done in a muffle furnace at $300{ }^{0} \mathrm{C}$ for $10 \mathrm{~min}$ 
391

392

393

394

395

396

397

398

399

400

401

402

403

404

405

406

407

408

409

410

411

412

413

414

under atmospheric conditions. The as produced black solid was collected and the final product was analyzed.

\subsection{Carbon nanotubes/carbon nanofibers}

One of the most systematically studied nanostructures, carbon nanotubes (CNTs) are cylindrically shaped materials with lengths in the order of few microns while the cross-sectional diameters are in the nanometer range $[106,107]$. Elongated surface of these materials makes them robustly versatile for their functionalization driven need based applications. Although the hybridization of constitutive carbon atoms is $\mathrm{sp}^{2}$ (similar to graphene), but the arrangement of atoms is relatively distinct (that does not form layers). The two known variations are single walled and multiwalled, with a high purity and cost of former. The most extraordinary feature of these materials is their structural toughness, imparted by inherently high rigidity, Young's Modulus, coefficient of elasticity which together are the reasons for their robust suitability in civil, defense, aeronautic and many other strategic applications $[107,108]$. It is because of such remarkable properties that these materials are widely preferred for developing immobilization based assays, with high detection sensitivities. An interesting aspect of these nanomaterials is that based on their geometry and chiral carbon vicinity, these can be electrically conductive, semi-conductive or insulated [107]. These adjustable electronic properties form the basis of their usage in single electronic transistors, flexible automated diodes where electron flow needs to be manipulated [109]. Comprised of only carbon, a variety of substrates have been used to obtain nanotubes via differently explored mechanisms. The most widely used methods of preparation are laser ablation, CVD [110], and electric discharge which necessitate the provision of specific stoichiometric mix of precursors. Though there are some concerns regarding the drug delivery application of these materials (with a potential risk of toxicity initiation), still the ability of functionalization has minimized such concerns and enabled a dose and location specific drug delivery with them. Readers are suggested 
415 to concern more specific literature sources regarding the biological applications of these

416 nanomaterials. Substrates as common as biscuits, chocolates, waste tyres, rubber and manifold

417 carbon comprising substances have been used to prepare carbon nanotubes [111-113].

$418 \quad 4.3$ CNTs from fly ash

419 Fly ash constitutes one of the most primitive by products, most widely produced through

420 pulverized coal combustion at the time of electricity generation in thermal power plants (TPPs)

421 [114]. Other than coal combustion, industrial activities such as mining and metallurgical

422 operations also contribute to fly ash generation. Compositionally, fly ash comprises of diverse

423 minerals and carbon materials either in single or combined form. The toxicity risk of fly ash has

424 recently been in news pertaining to deteriorating environmental quality in many developed and

425 developing nations across the globe. In these circumstances, a potential utilization of these

426 materials towards preparation of nanomaterials like CNTs [115], fullerenes [56] and several others

427 could be a significant breakthrough remedy to improve the pollution and toxicity extents and

428 contents of environment. Traces of carbon in the fly-ash are derived from organic contents and

429 incomplete combustion of coal, soots and charcoal combustion end products [116]. Several studies

430 report the preparation of CNTs from fly ash, with a 2016 study claimed the utility and aptness of

431 Saudi Arabian fly ash to provide CNTs using chemical vapor deposition method, provided all

432 reaction conditions are maintained [117]. The preparation of CNTs from fly ash could be

433 considered as an alternative to famous electric arc-discharge method, with significant reports of

434 transition metals ( $\mathrm{Mn}, \mathrm{Mg}, \mathrm{Ca}, \mathrm{Na}, \mathrm{Pb}, \mathrm{Cd}, \mathrm{Cr}, \mathrm{Co}, \mathrm{Ni}, \mathrm{Zn}$ and $\mathrm{Mo}$ ), present as traces in the fly ash.

435 Depending on the regional geography and parent source of generation, the transition metal

436 composition and diversity extents may vary amongst different sources. A generalized idea of

437 typical fly ash composition is mentioned in Table 1. This synthesis of CNTs serves dual purpose,

438 one being the minimization of hazardous waste in the environment while the other being the cost 
439 effectiveness and minimized use of energy. So, this approach is fittingly a green solution to

440 minimize the undesired environmental risks of fly-ash by means of sustainable approach. Research

441 on particulate matter pollution does pose a concern of significant respiratory complications from

442 inhaling fly-ash.

Table 1 Elemental composition of fly ashes

\begin{tabular}{|c|c|}
\hline Elements & Composition (wt. \%) \\
\hline $\mathrm{SiO}_{2}$ & $40-60 \%$ \\
\hline $\mathrm{Al}_{2} \mathrm{O}_{3}$ & $20-40 \%$ \\
\hline $\mathrm{Fe}_{2} \mathrm{O}_{3}-\mathrm{Fe}_{3} \mathrm{O}_{4}$ & $5-15 \%$ \\
\hline $\mathrm{TiO}_{2}$ & $2-5 \%$ \\
\hline Carbon & $5-20 \%$ \\
\hline $\mathrm{CaO}, \mathrm{BaO}, \mathrm{MgO}, \mathrm{MnO}, \mathrm{P}_{2} \mathrm{O}_{5}$ & Traces \\
\hline
\end{tabular}

446 Carbon based everyday gadgets, such as plastic materials, tyres [67], rubber end products and 447 several other forms can be readily used for making CNTs, using several modifications in their 448 subsequent chemical treatment approaches. The generation of plastic wastes to the tune of billion 449 tones on an everyday basis is one of the most pulsating concerns, since plastic wastes often 450 encounter a disposal problem due to their biodegradability concerns. Plastics are viciously 451 produced as waste products from industries, household routines, laboratories, hospitals and 452 eateries. Although the non-biodegradable nature of these materials has resulted in their substantial 453 recycling, but the recycled plastics often lose their plasticity. Many studies have nevertheless used 454 the plasticity intact waste materials to make CNTs via processing under varying oxygen 455 environments. In one such study, plastic waste was readily decomposed to propylene which 
456 subsequently catalyzed the MWCNT formation over the surface of metal catalysts [118]. Growth

457 mechanism is well known with reportedly following a tip-growth or base-growth pattern in course 458 of a vapor-liquid-solid reaction $[119,120]$. Nevertheless, there is still no clarity regarding the 459 utilization of carbon atoms whether in the bulk catalyst or react within the top surface of catalyst. 460 The reaction was mediated by the utilization of reactor material (SS 316 tube of a CVD reactor), 461 with the confirmatory studies revealing that removal of $\mathrm{Cr}$ from the reactor vessel resulted in 462 MWCNT growth. Similar studies on SS 316 mesh surface found an involvement of Fe and Ni in 463 the CNT formation. The results were in agreement of the works by Levendis and co-workers with 464 a further ensuring of metal catalyst prevalence along the tip of MWCNTs inside the tubes [121]. 465 Similarly, a 2016 study by Zhang and Williams reports the synthesis of MWCNTs along with 466 hydrogen generation by the catalytic pyrolysis of waste tyres. The study employed a catalyst 467 system comprising of a $\mathrm{Ni} / \mathrm{Al}_{2} \mathrm{O}_{3}$ prepared via impregnation of $\mathrm{Ni}$ on $\mathrm{Al}_{2} \mathrm{O}_{3}$ surface. The 468 experimental procedure was optimized by varying the temperature from (700 to 900$){ }^{0} \mathrm{C}$, alongside 469 varying the tyre to catalyst ratios from 1:0.5 to $1: 1$ and 1:2 and using steam input via injection of 470 hot water at 0,2 and $5 \mathrm{~mL}$ per hour injection rates. Estimation of the carbon fractions (formed as 471 product) revealed $253.7 \mathrm{mg}$ per gram tyre to be comprising of filamentous carbons at 1:1 tyre to 472 catalyst ratios at a catalyst temperature of $900{ }^{\circ} \mathrm{C}$. Microscopic screening of the product showed a 473 significant proportion of deposited filamentous carbons as MWCNTs. The procedure also released 474 hydrogen at compatible rates that met the fuel and energy scarcity, making this overall approach a 475 reliable an efficient methodology to utilize the tyre waste. An important aspect of this approach 476 was that it firstly processed the nickel nitrate as nickel precursor by its dissolution in ethanol on 477 alumina support that gradually converted into slurry via continuous stirring. Final catalyst was 478 prepared on overnight drying of this slurry at $90{ }^{0} \mathrm{C}$ in an oven, at $2{ }^{0} \mathrm{C}$ per minute till the 
479 temperature reached $750{ }^{\circ} \mathrm{C}$. This process took nearly a three hour holding time following which

480 the solid material collected was crushed into $(0.05-0.18) \mathrm{mm}$ sized granules. It is interesting to

481 note that the smaller size of catalyst particles conferred a higher surface area to the reacting species,

482 so whether a different physical form of the particles would be able to provide the product in same

483 morphology with a similar yield, remains a significant concern [113].

484 Quite recently, the synthesis of CNTs was reported from waste rubber based substrates, with the 485 experimental procedure utilizing the blended form of acrylonitrile butadiene and styrene butadiene 486 rubbers (NBR and SBR). The blend could not be conventionally decomposed due to its stronger 487 mechanical strength and thermal resistance, however the pyrolysis of the disposable form of blend 488 was optimized at $450{ }^{\circ} \mathrm{C}$ in a horizontal CVD pyrolyzer with a cautiously maintained nitrogen 489 supply to yield hydrocarbon fractions. Upon allowing the CVD of these hydrocarbon fractions on 490 different catalytic systems at $850{ }^{\circ} \mathrm{C}$ for half an hour, the screening of formed product using 491 HRTEM, Thermo gravimetric analysis (TGA) and Raman spectroscopy inferred a significant 492 formation of SWCNTs in an efficient extent. Subsequently, in course of physical analysis, it was 493 noted that adjusting the crystallinity of Fe-Ni catalyst on different zeolites was critical factor 494 affecting the structure and diameter of as formed CNTs [67]. So, approaches like these are all 495 potential solutions to synthesize nanotubes in desired yields from robust, cheap and biocompatible 496 materials ensuring minimal pollution risk and higher output yields compared to costly and energy 497 intensive conventional methods.

499 In the different parts of world, rice husk $(\mathrm{RH})$ shows as one of the most dominant crop residues 500 and the disposal of which often results in crucial environmental risks [122-124]. The major 501 constituent of $\mathrm{RH}$ as well as it burnt ash is silica (up to 90\%) (widely used as fillers and area 502 enhancement specific applications). So, efforts to utilize RH in its native as well burnt forms as 
503 reliable material providing energy are on a rapid high. Furthermore, the global RH production

504 registered a nearly $6 \%$ increase from 2010 to 2014 , which concerns with its alarming threat as 505 environmental hazard [125]. The utilization of rice husk (substantially comprising carbon, nitrogen

506 and hydrogen) commences with gasification (or pyrolysis), which generates fragments suitable for

507 power generation and biologically compatible charcoal. The one deemed fit for power generation 508 could be utilized as such via landfilling and fertilizer application. However, the fraction acting as 509 bio-reduced char, contributes significantly in industrial activities. This fraction provides three 510 potential materials, active carbon, porous carbon and amorphous silica, all of which have highly 511 good absorption characteristics conferred by their significant surface area contributions. While 512 amorphous silica finds peculiar suitability in soil improvement and cement industry the active and 513 porous carbon fractions are highly efficient adsorbents and used for waste water treatment 514 applications. So, with a carbon texture, the normally waste RH could be potentiated into manifold 515 useful industrial products. Readers can have a detailed look about the RH utilization and 516 processing methodology in a highly informative Nguyen et al contribution, reported in 2019 itself.

517 This is a review article that comprehensively discusses the engineering and industrial potential of $518 \mathrm{RH}$ and its derivative fractions (such as silicon nitride, magnesium silicide and others) as refractory 519 materials, filler agents in thermoplastics, as reinforcement agent, adsorbent in polymer composites 520 and many others.

\subsection{CNTs and graphene synthesis from oil}

522 Oils are one of most used commodities which are basically natural hydrocarbon precursors having 523 varying carbon chain fatty acids. The carbon skeleton of oils, accompanied by a range of physical 524 and chemical modifying technologies such as fractional crystallization, fractional distillation, 525 chromatographic separation, aqueous two phase attraction are the incentives for their reduction 526 procedures that could enable a range of products. Several kinds of oils, such as turpentine, 
527 eucalyptus, palm, turpentine, neem and sunflower, have been reported to enable efficient scale

528 synthesis yields of CNTs and graphene [126]. The use of turpentine oil in the making of CNTs has

529 been proposed by Chatterjee et al, through its decomposition on the surface of finely dispersed Co

530 catalyst at $675{ }^{\circ} \mathrm{C}$ optimized the CVD method to synthesize CNTs. The study also proposed the

531 application of synthesized CNTs in making efficient electrochemical double layer capacitor [127].

532 In several interesting modifications, scientists have optimized the use of neem, sunflower, sesame,

533 camphor and castor oils as the parent carbon sources for CNT synthesis. Utilization of sesame oil

534 has attracted significant scientific attention, owing to its edible nature, clean methodology and

535 formation of hollow CNTs with diverse shapes and morphology [128]. The formed nanotubes had

536 no Fe nanoparticles in the interior, had diameters within (50-60) nm and sheet-like structure

537 showing an intricate long-range array of folds. Thus, synthesis of nanotubes from oil represents

538 the renewable, energy efficient, cost effective and most importantly, much more compatible to

539 environment and laboratory personnel [129]. So, since the CNTs inception, making CNTs in big

540 yields is now no more a herculean task like in the beginning years.

\subsection{CNTs and graphene from poultry waste}

542 Poultry products or waste are also rigorous sources of carbon materials and their derivatives and 543 are mostly comprised of carbohydrates and proteins, along with a dense supplement in the form of 544 calcium [130]. Regarding the utilization of these materials to meet the energy concerns, egg shell 545 material promises to be a very rich source of providing carbon skeleton, it has been used with 546 significant interest to optimize the microbial growth for designated yields of biofuels. Though 547 CNTs are concretely not reported as being synthesized from these materials, yet a modified version, 548 namely, C-dots (inherently carbon comprising quantum dots) have successfully been synthesized 549 using this natural resource. Primary advantage of these nanomaterials compared to conventional quantum dots is their low toxicity. A 2012 study reported from China has optimized the microwave 
551 assisted approaches (providing intensive and efficient energy) to process egg shell material for a

552 reduced reaction time to obtain C-dots [131]. The study aimed at the microwave treatment of egg

553 shell material to form C-dots, having a maximum fluorescence peak (at $450 \mathrm{~nm}$ ) alongside a

554 quantum yield of $14 \%$. The modification of operational parameters like reaction time (microwave

555 duration), temperature, the relative contents of egg shell material could be the significant leads in

556 obtaining many other variations in the products, for obtaining the biologically and biophysically

557 more robust product designs.

\section{5. Applications of 2D nanomaterials}

559 The specialty of nanomaterials lies in their tunable nanoscale dimensionality, on the basis of which

560 these are considered as one, two or three dimensional [132]. Thus, two dimensional nanomaterials

561 are typically those materials which have two of their three dimensions restricted to $<100 \mathrm{~nm}$ [133].

562 There is not clear consensus regarding the upper limit of this restriction. This implies that in these

563 materials, it is feasible to retrieve the quantum scale effects on two dimensions, i.e. the restriction

564 of the electronic motions of excited state electrons (more conventionally known by the terminology

565 "quantum confinement"). The examples of these materials include nanosheets, fibrous networks

566 having nanometric widths and heights with lengths in the order of micrometers. Popular

567 applications of these materials include their inclusion as catalysts, electronic/battery devices,

568 hydrogen sensing, laser protection, magnetic memory devices and other domains, based on surface 569 plasmon resonance (SPR) attributes [134].

\subsection{Catalytic applications of 2D nanomaterials in fuel cells}

571 In the present day energy savvy scenario, everyone is anxious to obtain quicker and greater product

572 formation, minimizing not only the operational steps but also the energy requirements. 2D NMs

573 serve as ideal solutions to all these concerns in having a high aspect ratio, high electron mobility, 574 unsaturated surface coordination, and unique material properties (especially physical, chemical 
575 and electronic) [135]. The ultrafine thickness of these materials confers them with ultrahigh

576 specific surface areas and high surface energy, making them appropriate towards numerous surface

577 active applications such as for those in fuel cells. For efficient working of these cells, oxygen

578 generation and transport has to take place at reasonably good rates. The catalytic approaches in

579 most general cases employ platinum (Pt) nanoparticles (NPs) immobilized on the surface of carbon

580 substrate. However, due to their high costs and slow reaction kinetics, use of Pt NPs is not

581 economically as well as commercially viable. To tackle these issues, developments of new

582 methods like alloying and nanostructured engineering which could ensure a maximum activity,

583 stability along with cost minimization has emerged to be a priority [97]. Amongst the several

584 different shapes attainable by noble metal alloys, ultrathin 2D sheet like structures having a single

585 or few atoms thickness are acquiring significant interest because of their large size, high electron

586 mobility and surface energy. These features confer a high surface area to volume ratio to the

587 ultrathin 2D sheet like materials thereby giving rise to a high density of unsaturated atoms. For

588 instance, Hong et al have reported faster ethanol oxidation using ultrathin free standing Pd-Pt-Ag

589 (ternary) noble metal alloy [136]. Similarly, Din et al proposed a suitability of quaternary noble

590 metal alloy Pt-Cu-Bi-Mn (porous nanosheets) having (3 to 4) nm thickness as novel catalysts

591 having high oxygen (reduction and oxidation) capabilities apart from a significant methanol

592 tolerance [137].

593 Sial et al., have rigorously compiled the several methods of making nanosheets (NSs) and their

594 limitations in the present scenario (pertaining to energy considerations and economic constraints).

595 Different methods of synthesizing 2D NSs are carbon monoxide (CO) confined growth,

596 hydrothermal/solvothermal synthesis, wet chemical synthesis, self-assembly of NPs, topo

597 chemical reduction method, template based synthesis, seeded growth and microwave assisted 
598

599

600

601

602

603

604

605

606

607

608

609

610

611

612

613

614

615

616

617

618

619

620 growth. Well even though each of the methods provides specific characteristics of products in terms of morphology, the unanimous factors affecting their implementation are the need of robust catalysis (which offers lesser reaction time and is less costly) and the requirement of energy from external agency. For example, $\mathrm{CO}$ assisted growth method allows the preferential growth on the substrate due to a good surface adsorption of CO. These methods are workable through a feasibility of interactional distinctions of water and non-aqueous solvents, such as viscosity and dissociation constant. The process is characterized by selective oxidative etching enabling an attainment of specific anisotropic growth. Two critical requirements of these methods are optimum reaction temperature maintenance alongside the steady action of reducing agent. Likewise wet chemical synthesis offers layered patterns of ultrathin NSs, with industrially scalable products allowing no $\mathrm{CO}$ requirement (unlike the $\mathrm{CO}$ assisted growth method and hydrothermal/solvothermal method). Another mechanism of interest is self-assembly which provides NSs regulated by weak binding interactions and comparatively larger sizes. But the advantage in this method is that requirement of energy from external end is very low and the constituent species themselves acquire a minimum energy configuration. Likewise, the topo chemical reduction approach is specifically suited for making single crystalline metal alloy NS utilizing Ni and Co as combined catalyst in aqueous medium while template synthesis method is an efficient strategy to obtain layered nanostructures and extensively utilizes graphene and its derivatives as templates. Comparing the basic requirements of these two methods, it is quite evident that template synthetic approach offers much higher control with every successive step being regulated by the chemical composition of preceding deposited material layer. Another benign approach for making 2D NSs is the use of microwave technology, which is specially preferred for making inorganic nanomaterials having high quantum yield and high precision. Although this is green approach but yet again dependent 
621 on energy input from outside. Often template based synthesis mechanisms utilizing

622 hydrothermally fabricated catalysts are relied for commercial purposes.

623 The working of fuel cell involves rigorous electrochemical processes, characterized by 624 electrocatalytic oxygen reduction reaction (ORR) and hydrogen evolution reaction (HER), 625 involving formic acid oxidation and alcoholic oxidation at cathode and anode. The major problems 626 encountered in commercialized application of fuel cells are improvements in the electrode 627 preparation with minimized use of precious metals, controlling the kinetics of electrochemical 628 process which collectively reduces the output efficiency of a fuel cell. So, in general faster, more 629 efficient and rigorous catalysis with minimized expenditure and care requirements are the key. 630 With continuous better understanding, several alternative mechanisms have emerged as steady 631 sources of energy provision, like microbial driven fuel cells which utilize the energy generated 632 from microbial metabolism (the functioning of enzymes and key pathways). However, this 633 recourse is also not free of constraints as there is a constant need to ensure optimum microbial 634 activities through providing specific $\mathrm{pH}$, temperature, humidity and minimizing the ion 635 concentration [138]. Recently, a new methodology making use of CNT based composite materials 636 have emerged. The concurrent hindrances related to dependence on water for conductivity, high 637 methanol permeability, frequent disintegration (of conventionally used materials) in the presence 638 of $-\mathrm{OH}$ radicals and low to moderate chemical stability have been the reasons to screen a safer, 639 more reliable and efficient alternative. A novel attempt in this direction has been the use of nafion 640 based membranes and its composite with inclusion of CNTs as polymer electrolyte material (PEM) 641 has provided a solution to recurrent limitations, through its greater mechanical stability, greater 642 tensile strength and stronger physical texture [139]. Thus, nanomaterials provide numerous 
643 structural benefits to improve the fuel cell working through improvement in catalysis and energy

644 savvy functioning.

645

646

5.2. Applications related to surface plasmon resonance

647 more specifically the metal or metal oxide NPs. These entities absorb light in maximum at a

648 peculiar wavelength after which the constituent ions are excited and progressively move to high

649 energy state. As the temperature increases (due to the input heat or light energy or via

650 intermolecular frictional activities), these excited particles rapidly move with a net charge and

651 remain in the semi-solid state, termed as plasma. The terminology plasmon is originated from the

652 essence of ions existing in plasmonic state. The resonance implies an instant where the light energy

653 absorption is maximum, owing to which the manifested surface effects are also greater. Each

654 nanoparticle has a characteristic SPR corresponding to peculiar kind of incident light, so the SPR

655 wavelengths are often used as identifiers for the formation of specific NPs. Since there is maximum

656 energy absorbance in the SPR event, so the nanoscale effects are also highest at this particular

657 instant, giving rise to maximum bioactivities or quantum confinement dependent properties. The

658 applications of nanomaterials have been significantly improved after a clear understanding of this

659 phenomenon, with bulk species or sensing moieties being swiftly replaced either by individual

660 NPs (bound in membranes) or by combination of nanomaterials (such as assembled nanostructures

661 or hybrid NPs and thin layers of nanomaterials. For detailed insights of SPR and its consequent

662 applications, readers are suggested to refer more specific literature contribution [140].

663

\subsection{Nanotechnology and solar energy}

664 Probably, the most clean, unanimously accessible and even most used form of energy, the solar 665 energy is a rigorous input agency for most of the daily life activities. From microbes to plants, 666 animals and even human beings, all require solar energy directly or indirectly for sustenance of 
667 life. Commercial usage of solar energy presents exciting prospects, which are often limited by its 668 low efficiency (substantially attributed to uncertainty of availability) and inabilities to being scaled 669 up. Lots of progress has been made via use of nanomaterials in native and engineered form, to 670 increase the absorption efficacy of sun's energy radiations. Most popular area has been the use of 671 solar cell panels to provide electricity in which the functional circuit comprises of an assembly of 672 solar cells in a rectangular pattern. The efficiency of original assembly is quite low owing to which 673 Si wafers (with amicable impurities) are added to it, which collectively not only improve the 674 absorption but also manifold the utilization extents. Similarly, nanoscale attenuators and 675 converters have been drafted into calculators to improve their charging efficiencies and 676 performance. Lots of bioassays and drug carrier systems are in the market working through 677 photothermal attributes of metallic NPs and their constitutive assemblies. Thin layers or assemblies 678 of nanomaterials have emerged as carriers of more uniform and regulated solar energy absorption 679 that remain localized to the surface and do not cause any serious effect in the bulk. Piezoelectric 680 materials (such as $\mathrm{MgO}$ and $\mathrm{ZnS}$ based nanostructures) have come to the forefront, making use of 681 pressure influences from solar energy (as input) to conduct the electricity or perform mechanical 682 works. Many of these conceptualizations are in the research phase with delay in optimization 683 studies, meeting the scale-up regulations and constraints; owing to which commercialization of 684 such innovations is being delayed. Considering the energy crisis scenario (in particular for 685 developing world), these solutions could be potential remedies to eradicate the inadequate energy 686 availability. Recently, the use of nanofluids (typically having either solid NPs or (1-100) nm sized 687 nanofibers suspended in a liquid) has been on peculiar rise to enhance the utilization potential of 688 solar energy [141]. These fluids having dissolved nanomaterial(s), are able to enhance the outlet 689 temperature by (30-100) $\mathrm{K}$, enabling an enhanced potential to absorb the sunlight without any 
690 damage to native structures of base material. One study claimed more than $100 \%$ enhancement in

691 photo thermal efficiency of $0.01 \%$ graphite based nanofluid than without using it (normal

692 functioning mode involving coating of an absorbing collector). The use of these fluidic materials

693 has enabled improved photovoltaic application via long lasting existence in non-agglomerated

694 form, having high stability without undergoing significant chemical changes in base fluid [142].

695 The use of nanofluids has significantly improved the efficiency of electrolysis manifolds by the 696 replacement of conventional electrolytes, allowing faster and smoother conduct of chemical

697 reactions [143].

698 6. Conclusions

699 The progress and better understanding of nanotechnology and its functional principles have slowly 700 entered into the multiple inter and cross-domain disciplines, to improve the product life, design, 701 performance and overall quality by considerable reduction in the raw materials. The use of 702 nanostructures like CNTs, 2D nanosheets, several different kinds of NPs has provided stronger 703 and more efficient materials, enabling multifunctional performance and increased outputs. 704 Incorporation of CNTs along with graphene and their derivatives have bettered the present 705 performance of materials by substantial improvement in their structural responses, mechanical 706 strength, stress bearing capacity and physical load bearing capacities. The availability of these 707 materials in multiple nanoscale dimensions has enabled the synthesis of desired materials with 708 robust self-adjusting responses and flexibilities. Though much remains unknown and even 709 unpredictable that sometimes poses a risk in their nanoscale manifested enhanced chemical 710 reactivity, still regular research and continuous merger of scientific cross disciplines have 711 significantly improved the understanding with respect to use of nanomaterials. Market scenario 712 predicts hopeful aspects from consumer point of viewpoint while at the same time, appearing little 
713 gloomy for the reduced manpower requirement. So, better understanding of nanomaterials usage

714 and applications definitely owes a bright future and better living standard for mankind.

715

716

\section{References}

717 1. Mansoori, G.A. and T.A.F. Soelaiman, Nanotechnology-an introduction for the standards. 718

719 2. Malshe, A., Nanotechnology. 2016: p. 1-9.

720 3. Lošdorfer Božič, A., A. Šiber, and R. Podgornik, Statistical analysis of sizes and shapes of 721 virus capsids and their resulting elastic properties. Journal of biological physics, 2013. $722 \quad 39(2):$ p. 215-228.

723 4. Mansoori, G.A., Advances in Atomic and Molecular Nanotechnology. 2005.

724 5. Ali, S., et al., Synthesis of $\gamma$-alumina $\left(\mathrm{Al}_{2} \mathrm{O}_{3}\right)$ nanoparticles and their potential for use as 725 726 727 728 729 730

7. Hanemann, T. and D.V. Szabó, Polymer-Nanoparticle Composites: From Synthesis to an adsorbent in the removal of methylene blue dye from industrial wastewater. Nanoscale Advances, 2019. 1(1): p. 213-218.

6. Pyun, J. and K. Matyjaszewski, Synthesis of Nanocomposite Organic/Inorganic Hybrid

7349 9. A., Chapter - INTRODUCTION TO NANOMATERIALS. 2011. p. 76.

735 10. Rana, S., et al., Nanomaterials from Natural Products for Industrial Applications. Vol. 736 2017. 2017. 1-2.

737 11. Dutta, T., et al., Recovery of nanomaterials from battery and electronic wastes: A new 738 paradigm of environmental waste management. 2017.

739 12. Ali, H. and M. Hassaan, Applications of Bio-waste Materials as Green Synthesis of 740 Nanoparticles and Water Purification. Vol. 6. 2017. 85-101. 
741 13. Banerjee, P. and D. Nath, PLANTS AND ECO-FRIENDLY NANOMATERIALS-A 742 REVIEW. 2015.

743 14. Yadav, V.K. and M.H. Fulekar, Isolation and charcterization of iron nanoparticles from 744 coal fly ash from Gandhinagar Gujarat thermal power plant a mechanical-method-of745 746 isolation. INTERNATIONAL JOURNAL OF ENGINEERING RESEARCH \& TECHNOLOGY (IJERT), 2014. 3(6): p. 471-477.

747

15. Sangeetha, J., et al., Production of Bionanomaterials from Agricultural Wastes. 2017. p. 748 33-58.

749

16. Zheng, Y., et al., Synthesis of Carbon Nanotube Arrays with High Aspect Ratio via NiCatalyzed Pyrolysis of Waste Polyethylene. Nanomaterials, 2018. 8(7): p. 556.

17. Kandil, M., THE ROLE OF NANOTECHNOLOGY IN ELECTRONIC PROPERTIES OF 752 MATERIALS. 2016.

19. Nikalje, A., Nanotechnology and its Applications in Medicine. Vol. 5. 2015. 5:081-089.

20. Jeevanandam, J., et al., Review on nanoparticles and nanostructured materials: History, 758 sources, toxicity, and regulations. Vol. 9. 2018. 1050-1074.

21. Edvinsson, T., Optical quantum confinement and photocatalytic properties in two-, oneand zero-dimensional nanostructures. Royal Society Open Science. 5(9): p. 180387.

22. Brehm, M. and M. Grydlik, Site-controlled and advanced epitaxial Ge/Si quantum dots: fabrication, properties, and applications. Nanotechnology, 2017. 28(39): p. 392001.

23. Patel, K.D., R.K. Singh, and H.-W. Kim, Carbon-based nanomaterials as an emerging platform for theranostics. Materials Horizons, 2019. 6(3): p. 434-469.

24. Singh, R. and R. Singh, A Review on Nano Materials of Carbon. Vol. 9. 2017. 42-57.

25. Han, N., F. Wang, and J. Ho, One-dimensional nanostructured materials for solar energy harvesting. Vol. 1. 2012. 4-17.

26. Om, K. and K. Manjit, Single Electron Transistor: Applications \& Problems. Vol. 1. 2010.

27. Cao, H., Synthesis, Characterization, and Applications of Zero-Dimensional (OD) Nanostructures. Synthesis and Applications of Inorganic Nanostructures, 2017. 
771 28. Tiwari, J.N., R.N. Tiwari, and K.S. Kim, Zero-dimensional, one-dimensional, two772 dimensional and three-dimensional nanostructured materials for advanced 773

30. Edelstein, A.S., Nanomaterials, in Encyclopedia of Materials: Science and Technology, K.H.J. Buschow, et al., Editors. 2001, Elsevier: Oxford. p. 5916-5927.

31. Whitby, R.L.D., et al., 1D Nanomaterials. Journal of Nanomaterials, 2010. 2010.

779

32. Zhao, Y., et al., ID Nanomaterials: Synthesis, Properties, and Applications. Vol. 2013. 2013.

33. Fang, X. and L. Zhang, One-Dimensional (1D) ZnS Nanomaterials and Nanostructures. Vol. 22. 2006.

34. Zhang, C., et al., Synthesis and applications of organic nanorods, nanowires and nanotubes. Vol. 109. 2013. 211-239.

35. Guo, Z. and L. Tan, Fundamental and applications of Nanomaterials. 2009, ARTECH HOUSE: 685 Canton Street

Norwood. p. 1-241.

36. Zhang, Z., et al., One-dimensional silicon-based semiconductor nanomaterials: synthesis, structures, properties and applications. Vol. 36. 2011. 148-173.

37. Gnanamoorthy, G., et al., Enhanced photocatalytic performance of $\mathrm{ZnSnO3/rGO}$ nanocomposite. Chemical Physics Letters, 2020. 739: p. 137050.

39. Klein, E., R. Lesyuk, and C. Klinke, Insights into the formation mechanism of twodimensional lead halide nanostructures. Nanoscale, 2018. 10(9): p. 4442-4451.

40. Sahoo, P.K., et al., Recovery of metals and other beneficial products from coal fly ash: a sustainable approach for fly ash management. International Journal of Coal Science \& Technology, 2016. 3(3): p. 267-283. 
801 41. Yin, P.T., et al., Design, synthesis, and characterization of graphene-nanoparticle hybrid 802 803

42. Lin, J., X. Chen, and P. Huang, Graphene-based nanomaterials for bioimaging. Advanced 804 805

43. Shah, M., et al., Green Synthesis of Metallic Nanoparticles via Biological Entities. 806 Materials, 2015. 8(11): p. 7278-7308.

807

44. Koski, K.J. and Y. Cui, The New Skinny in Two-Dimensional Nanomaterials. ACS Nano, 808 2013. 7(5): p. 3739-3743.

809

45. Gnanamoorthy, G., et al., Implementation of $\mathrm{ZnSnO}_{3}$ nanosheets and their $\mathrm{RE}$ (Er, Eu, and 810 Pr) materials: Enhanced photocatalytic activity. Advanced Powder Technology, 2020. 31(3): p. 1209-1219.

46. Boroumand Moghaddam, A., et al., Nanoparticles Biosynthesized by Fungi and Yeast: A

815 47. Miró, P., M. Audiffred, and T. Heine, An atlas of two-dimensional materials. Vol. 43. 2014.

816 48. Van Gough, D., A.T. Juhl, and P.V. Braun, Programming structure into 3D nanomaterials. 817 Materials Today, 2009. 12(6): p. 28-35.

818 49. Yaya, A., Layered Nanomaterials-A Review. Vol. 1. 2012. 32-41.

819 50. Sharma, S., et al., Nanostructured Materials for Food Applications: Spectroscopy, $820 \quad$ Microscopy and Physical Properties. Bioengineering, 2019. 6(1): p. 26.

821 51. Wang, J. and H. Gu, Novel Metal Nanomaterials and Their Catalytic Applications. 822 Molecules, 2015. 20(9): p. 17070-17092.

823 52. Julien, M.C. and A. Mauger, Nanostructured MnO2 as Electrode Materials for Energy 824

825 53. Singh, P. and S. Jain, Biosynthesis of Nanomaterials: Growth and Properties. Vol. 3. 2014. Storage. Nanomaterials, 2017. 7(11). $826 \quad 231-238(8)$.

827 54. Luther, W., Industrial Application of Nanomaterials-Chances and Risks. Vol. 54. 2004. 828 55. Gavagnin, M., et al., Free-Standing Magnetic Nanopillars for 3D Nanomagnet Logic. ACS 829 Applied Materials \& Interfaces, 2014. 6(22): p. 20254-20260. 
830 56. Georgakilas, V., et al., Broad Family of Carbon Nanoallotropes: Classification, Chemistry, 831 and Applications of Fullerenes, Carbon Dots, Nanotubes, Graphene, Nanodiamonds, and 832 Combined Superstructures. Chemical Reviews, 2015. 115(11): p. 4744-4822.

833 57. Semaltianos, N.G., Nanoparticles by Laser Ablation. Critical Reviews in Solid State and 834 Materials Sciences, 2010. 35(2): p. 105-124.

835 58. Sarkar, J.K. and Q. Wang, Different Pyrolysis Process Conditions of South Asian Waste 836 Coconut Shell and Characterization of Gas, Bio-Char, and Bio-Oil. Energies, 2020. 13(8): 837 p. 13.

838 59. Dhand, C., et al., Methods and Strategies for the Synthesis of Diverse Nanoparticles and 839 840 841 842 843 844 845 846 847 848 849 850

60. Umadevi, S., R. Umamaheswari, and V. Ganesh, Lyotropic liquid crystal-assisted Their Applications: A Comprehensive Overview. Vol. 5. 2015. synthesis of micro- and nanoparticles of silver. Liquid Crystals, 2017. 44(9): p. 1409-1420.

61. Tonelli, D., E. Scavetta, and I. Gualandi, Electrochemical Deposition of Nanomaterials for Electrochemical Sensing. Sensors, 2019. 19(5): p. 1186.

62. Tierno, P. and W.A. Goedel, Using Electroless Deposition for the Preparation of Micron Sized Polymer/Metal Core/Shell Particles and Hollow Metal Spheres. The Journal of Physical Chemistry B, 2006. 110(7): p. 3043-3050.

63. Hayashi, H. and Y. Hakuta, Hydrothermal Synthesis of Metal Oxide Nanoparticles in Supercritical Water. Materials, 2010. 3(7): p. 3794-3817.

64. Li, J., Q. Wu, and J. Wu, Synthesis of Nanoparticles via Solvothermal and Hydrothermal Methods, in Handbook of Nanoparticles, M. Aliofkhazraei, Editor. 2016, Springer International Publishing: Cham. p. 295-328.

65. Ramesh, S., Sol-Gel Synthesis and Characterization of Nanoparticles. Journal of Nanoscience, 2013. 2013: p. 8.

66. Kumar Yadav, V. and M.H. Fulekar, Green synthesis and characterization of amorphous silica nanoparticles from fly ash. Materials Today: Proceedings, 2019. 18(Part 7): p. 43514359.

67. Essawy, H., et al., Fabrication of single-walled carbon nanotubes from vulcanized scrap rubber via thermal chemical vapor deposition. RSC Advances, 2017. 7(21): p. 1293812944. 
860 68. Piriyawong, V., et al., Preparation and Characterization of Alumina Nanoparticles in 861 Deionized Water Using Laser Ablation Technique. Journal of Nanomaterials, 2012. 2012: 862 p. 1-6.

863

69. Yadav, D.A., Synthesis of nanomaterials by physical and chemical methods Intenational 864 865 education \& research journal 2017. 3(6): p. 350-352.

866

867

71. Peralta-Videa, J.R., et al., Plant-based green synthesis of metallic nanoparticles: scientific curiosity or a realistic alternative to chemical synthesis? Nanotechnology for Environmental Engineering, 2016. 1(1): p. 4.

870 72. Aftab, A., Synthesis of Nanoparticles by Microbes. 2018. p. 175-193.

871 73. Castro, L., et al., Biological synthesis of metallic nanoparticles using algae. Vol. 7. 2013. $872 \quad 109-16$.

873 74. Iravani, S., Bacteria in Nanoparticle Synthesis: Current Status and Future Prospects. 874 International Scholarly Research Notices, 2014. 2014: p. 18.

75. Sharma, D., S. Kanchi, and K. Bisetty, Biogenic synthesis of nanoparticles: A review. Arabian Journal of Chemistry, 2015.

76. Kim, S., K.H. Kim, and C.W. Bark, Two-Dimensional Nanomaterials: Their Structures, Synthesis, and Applications. Science of Advanced Materials, 2017. 9(9): p. 1441-1457.

78. Colson, P., C. Henrist, and R. Cloots, Nanosphere Lithography: A Powerful Method for the Controlled Manufacturing of Nanomaterials. Journal of Nanomaterials, 2013. 2013(5).

79. Pingali, K.C., S. Deng, and D.A. Rockstraw, Synthesis of Nanowires by Spray Pyrolysis. Journal of Sensors, 2009. 2009: p. 6.

80. Suryanarayana, C. and B. Prabhu, Synthesis of Nanostructured Materials by Inert-Gas Condensation Methods. 2007. p. 47-90.

81. Habiba, K., et al., Fabrication of Nanomaterials by Pulsed Laser Synthesis. 2014. p. 263291.

82. Xu, H., B.W. Zeiger, and K.S. Suslick, Sonochemical synthesis of nanomaterials. Chemical Society Reviews, 2013. 42(7): p. 2555-2567. 
891 83. Ray, P.C., H. Yu, and P.P. Fu, Toxicity and Environmental Risks of Nanomaterials:

892

893

894

895

896

897

898

899

900

901

902

903

904

905

906

907

908

909

910

911

912

913

914

915

916

917

918

919

920

921
Challenges and Future Needs. Journal of Environmental Science and Health, Part C, 2009. 27(1): p. 1-35.

84. Gnanamoorthy, G., et al., New construction of $\mathrm{Fe}_{3} \mathrm{O}_{4} / \mathrm{rGO} / \mathrm{ZnSnO} \mathrm{O}_{3}$ nanocomposites enhanced photoelectro chemical properties. Optical Materials, 2020. 109(November 2020): p. 110353.

85. Yang, J., et al., Nanomaterials for the Removal of Heavy Metals from Wastewater. Nanomaterials, 2019. 9(3): p. 39.

86. Dai, Z.R., Z.W. Pan, and Z.L. Wang, Growth and Structure Evolution of Novel Tin Oxide Diskettes. Journal of the American Chemical Society, 2002. 124(29): p. 8673-8680.

87. Cha, C., et al., Carbon-based nanomaterials: multifunctional materials for biomedical engineering. ACS nano, 2013. 7(4): p. 2891-2897.

88. Savjani, K.T., A.K. Gajjar, and J.K. Savjani, Drug solubility: importance and enhancement techniques. ISRN pharmaceutics, 2012. 2012: p. 195727-195727.

89. Dubé, M.A. and S. Salehpour, Applying the Principles of Green Chemistry to Polymer Production Technology. Macromolecular Reaction Engineering, 2014. 8(1): p. 7-28.

90. Giurlani, W., et al., Electroplating for Decorative Applications: Recent Trends in Research and Development. Coatings, 2018. 8(8): p. 260.

91. Rai, M., et al., Strategic role of selected noble metal nanoparticles in medicine. Vol. 19. 2015. 1-24.

92. Khodashenas, B. and H. Ghorbani, Synthesis of silver nanoparticles with different shapes. Vol. 7. 2015.

93. Jiang, X., et al., Synthesis and growth of hematite nanodiscs through a facile hydrothermal approach. Vol. 12. 2009. 877-893.

94. Fu, J. and S.E. Skrabalak, Aerosol synthesis of shape-controlled template particles: a route to Ta3N5 nanoplates and octahedra as photocatalysts. Journal of Materials Chemistry A, 2016. 4(21): p. 8451-8457.

95. Swarnavalli, G.C.J., et al., A Simple Approach to the Synthesis of Hexagonal-Shaped Silver Nanoplates. Journal of Nanomaterials, 2011. 2011: p. 5.

96. He, X., et al., Shape-controlled synthesis for silver: Triangular/hexagonal nanoplates, chain-like nanoplate assemblies, and nanobelts. Vol. 24. 2009. 2200-2209. 
922 97. Zeb Gul Sial, M.A., M.A. Ud Din, and X. Wang, Multimetallic nanosheets: synthesis and

923

924

925

926

927

928

929

930

931

932

933

934

935

936

937

938

939

940

941

942

943

944

945

946

947

948

949

950

applications in fuel cells. Chemical Society Reviews, 2018. 47(16): p. 6175-6200.

98. Zhang, Z., et al., Sodium Citrate: A Universal Reducing Agent for Reduction/Decoration of Graphene Oxide with Au Nanoparticles. Vol. 4. 2011. 599-611.

99. Zhou, Y., et al., Synthesis of Metal Oxide Nanosheets through a Novel Approach for Energy Applications. Vol. 4. 2015.

100. Liu, J., H. Yang, and X. Xue, Preparation of different shaped $\alpha-F e 2 O 3$ nanoparticles with large particle of iron oxide red. Vol. 21. 2018.

101. Yadav, V.K., et al., Microbial Synthesis of Nanoparticles and Their Applications for Wastewater Treatment, in Microbial Biotechnology: Basic Research and Applications, J. Singh, et al., Editors. 2020, Springer Singapore: Singapore. p. 147-187.

102. Tiwari, S., et al., Magical Allotropes of Carbon: Prospects and Applications. Critical Reviews in Solid State and Material Sciences, 2015. 41(4): p. 257-317

103. Zaytseva, O. and G. Neumann, Carbon nanomaterials: Production, impact on plant development, agricultural and environmental applications. Chemical and Biological Technologies in Agriculture, 2016. 3(17).

104. Loh, Y.R., et al., Sugarcane bagasse-The future composite material: A literature review. Resources, Conservation and Recycling, 2013. 75: p. 14-22.

105. $\mathrm{Xu}, \mathrm{Q}$., et al., Characteristics and Applications of Sugar Cane Bagasse Ash Waste in Cementitious Materials. Materials (Basel), 2018. 12(1): p. 19.

106. Kroto, H.W., Heath, J.R., O'Brien, S.C., Curl, R.F., \& Smalley, R.E., buckminsterfullerene. nature, 1985. 318: p. 162-163.

107. Iijima, S., Helical Microtubules of Graphitic Carbon. Nature, 1991. 354: p. 56-58.

108. Byrne, M.T. and Y.K. Gun'ko, Recent advances in research on carbon nanotube-polymer composites. (1521-4095 (Electronic)).

109. Arora, N. and N.N. Sharma, Arc Discharge synthesis of Carbon Nanotubes: Comprehensive review. Vol. 50. 2014.

110. Vallejos, S., et al., Chemical Vapour Deposition of Gas Sensitive Metal Oxides. Chemosensors, 2016. 4(1): p. 4. 
951 111. Tripathi, P.K., S. Durbach, and N.J. Coville, Synthesis of Multi-Walled Carbon Nanotubes

952

953

954

955

956

957

958

959

960

961

962

963

964

965

966

967

968

969

970

971

972

973

974

975

976

977

978

979

980

981 from Plastic Waste Using a Stainless-Steel CVD Reactor as Catalyst. Nanomaterials (Basel, Switzerland), 2017. 7(10): p. 284.

112. Nguyen, H., M. Jamali moghadam, and H. Moayedi, Agricultural wastes preparation, management, and applications in civil engineering: a review. 2019.

113. Zhang, Y. and P.T. Williams, Carbon nanotubes and hydrogen production from the pyrolysis catalysis or catalytic-steam reforming of waste tyres. Journal of Analytical and Applied Pyrolysis, 2016. 122: p. 490-501.

114. Yadav, V.K. and M.H. Fulekar, The current scenario of thermal power plants and fly ash production and utilization: with a focus in India International Journal of Advance Engineering and Research Development, 2018. 5(4): p. 768-777.

115. Salah, N., et al., Formation of Carbon Nanotubes from Carbon Rich Fly Ash: Growth Parameters and Mechanism. Materials and Manufacturing Processes, 2015. 31: p. 150811005209005.

116. Hower, J., et al., Coal-derived unburned carbons in fly ash: A review. Vol. 179. 2017.

117. Salah, N., et al., Formation of Carbon Nanotubes from Carbon-Rich Fly Ash: Growth Parameters and Mechanism. Materials and Manufacturing Processes, 2016. 31(2): p. 146156.

118. Ramirez, A., et al., Unraveling the growth of vertically aligned multi-walled carbon nanotubes by chemical vapor deposition. Vol. 1. 2014. 045604.

119. Nessim, G.D., Properties, synthesis, and growth mechanisms of carbon nanotubes with special focus on thermal chemical vapor deposition. Nanoscale, 2010. 2(8): p. 1306-1323.

120. J. MacKenzie, K., O. M. Dunens, and A. T. Harris, An Updated Review of Synthesis Parameters and Growth Mechanisms for Carbon Nanotubes in Fluidized Beds. Vol. 49. 2010.

121. Zhuo, C., et al., Oxidative heat treatment of 316L stainless steel for effective catalytic growth of carbon nanotubes. Vol. 313. 2014. 227-236.

122. Azadi, M., M. Bahrololoom, and F. Heidari, Enhancing the mechanical properties of an epoxy coating with rice husk ash, a green product. Vol. 8. 2011. 117-123.

123. Yalçin, N. and V. Sevinç, Studies on silica obtained from rice husk. Vol. 27. 2001. 219224. 
982 124. Yalçın, N. and V. Sevinç, Studies of the surface area and porosity of activated carbons 983 prepared from rice husks. Vol. 38. 2000. 1943-1945.

125. FAO Statistical yearbook 2013: world food and agriculture, in Food and Agriculture Organization of the United Nations. FAO Fiat Fanis: Rome. p. 189.

126. Kumar, D.R., D.R. Singh, and D. Singh, Natural and waste hydrocarbon precursors for 988 989 990

130. Titirici, M., et al., Sustainable carbon materials. Chemical Society reviews, 2014. 44(1): p. 250-290.

131. Wang, Q., et al., Microwave-assisted synthesis of carbon nanodots through an eggshell membrane and their fluorescent application. Vol. 137. 2012. 5392-7.

132. Jeevanandam, J., et al., Review on nanoparticles and nanostructured materials: history, sources, toxicity and regulations. Beilstein journal of nanotechnology, 2018. 9: p. 10501074.

134. Alberto, N., et al., Optical Fiber Magnetic Field Sensors Based on Magnetic Fluid: A Review. Sensors (Basel), 2018. 18(12): p. 27.

135. Khan, K., et al., Recent Progress, Challenges, and Prospects in Two-Dimensional PhotoCatalyst Materials and Environmental Remediation. Nano-Micro Letters, 2020. 12(1): p. 77.

136. Hong, J.W., et al., Ultrathin Free-Standing Ternary-Alloy Nanosheets. Angewandte Chemie, 2016. 128(8): p. 2803-2808. 
1013 137. Ud Din, M.A., et al., Porous Tetrametallic PtCuBiMn Nanosheets with a High Catalytic 1014 Activity and Methanol Tolerance Limit for Oxygen Reduction Reactions. Advanced 1015 Materials, 2017. 29(8): p. 1604994.

1016 138. Zhao, F., J.R. Slade Rc Fau - Varcoe, and J.R. Varcoe, Techniques for the study and 1017 development of microbial fuel cells: an electrochemical perspective. Chem. Soc. Rev., 1018 2009. 38(0306-0012 (Print)): p. 1926-1939

1019 139. Kannan, R., B.A. Kakade, and V.K. Pillai, Polymer Electrolyte Fuel Cells Using Nafion1020 Based Composite Membranes with Functionalized Carbon Nanotubes. Angewandte 1021 Chemie International Edition, 2008. 47(14): p. 2653-2656.

1022 140. Huang, X., et al., Plasmonic PTT therapy (PPTT) using gold nanoparticles. Lasers Med 1023 Sci. Vol. 23. 2008. 217-28.

1024 141. Luo, Z., et al., Performance improvement of a nanofluid solar collector based on direct 1025 absorption collection (DAC) concepts. Vol. 75. 2014. 262-271.

1026 142. Thaker, R. and J. Patel, Application of Nano fluids in Solar Energy. Vol. 6. 2015. 1-11.

1027 143. Elsheikh, A.H., et al., Applications of nanofluids in solar energy: A review of recent 1028 advances. 2017.

1029

1030 